Received: 23.10 .2019

Revised: 29.11.2019

Accepted: 20.12 .2019

DOI: $10.17804 / 2410-9908.2019 .6 .026-036$

\title{
DETERMINATION OF THE ADHESIVE STRENGTH OF A LAMINATED GLUE MATERIAL UNDER THREE-POINT BENDING
}

\author{
S. V. Smirnov ${ }^{1, a^{*}}$, I. A. Veretennikova ${ }^{1, \text { b) }}$, D. I. Vichuzhanin ${ }^{1, c)}$, and A. V. Pestov ${ }^{2, d)}$ \\ ${ }^{1}$ Institute of Engineering Science, Ural Branch of the Russian Academy of Sciences, \\ 34 Komsomolskaya St., 620049, Ekaterinburg, Russian Federation \\ ${ }^{2}$ I. Ya. Postovsky Institute of Organic Synthesis, Ural Branch of the Russian Academy of Sciences, \\ 22 S. Kovalevskoy St., 620049, Ekaterinburg, Russian Federation \\ a) iD https://orcid.org/0000-0002-2083-5377 svs@imach.uran.ru;
b) iD https://orcid.org/0000-0002-8371-7546 irincha@imach.uran.ru;
c) iD https://orcid.org/0000-0002-6508-6859 mmm@imach.uran.ru;
d) iD https://orcid.org/0000-0002-4270-3041 mestov@ios.uran.ru \\ *Corresponding author. E-mail: svs@imach.uran.ru \\ Address for correspondence: 34 Komsomolskaya St., 620049, Ekaterinburg, Russian Federation \\ Tel.: +7 (343) 3744076
}

The test procedure and the results of testing the effect of stress on the adhesive strength of a glue joint under shear are discussed. Three-point bending testing of samples of an AMg6-NVP aluminum-magnesium multilayer alloy and a two-component cold-curing epoxy glue is chosen as a research method. An experimental correlation between the specific work of shear-type adhesive failure and the stress state has been obtained from video analysis and finite element modeling. It is shown that low adhesive strength is observed in the presence of tensile stresses normal to the joint plane. The work spent on shear-type adhesive failure intensively increases under the action of compressive normal stresses. The results can be used to evaluate the structural strength of adhesive joints under a complex stress state.

Keywords: adhesive failure, glue joint, epoxy glue, three-point bending test.

\section{Acknowledgment}

The experimental procedures for testing polymer coatings were developed in accordance with the research plan for the IES UB RAS, theme AAAA-A18-118020790145-0. The Ural Branch of the RAS financially supported (project 18-11-1-11) the purchase of the test materials and the three-point bending study of the effect of the stress state on the specific work of shear-type adhesive failure for a glue joint. The equipment installed in the Plastometriya collective use center, IES UB $R A S$, was used in the experimental investigation.

\section{References}

1. Hutchinson J.W., Suo Z. Mixed Mode Cracking in Layered Materials. Advanced in Applied Mechanics, 1992, vol. 29, pp. 63-191. DOI: 10.1016/S0065-2156(08)70164-9.

2. Volinsky A.A., Moody N.R., Gerberich W.W. Interfacial toughness measurements for thin films on substrates. Acta Materialia, 2002, vol. 50, pp. 441-466. DOI: 10.1016/S13596454(01)00354-8.

3. Tamuzh V.P., Protasov V.D. Razrushenie konstruktsiy iz kompozitnykh materialov [The Fracture of Structures Made of Composite Materials]. Riga, Zinatne Publ., 1986, 264 p. (In Russian). 
4. Tarnopol'skij Yu.M., Kincis T.Ya. Metody staticheskikh ispytaniy armirovannykh plastikov [Methods of Static Testing of the Reinforced Plastics]. Moscow, Khimiya Publ., 1981, 272 p. (In Russian).

5. Smirnova E.O., Veretennikova I.A., Smirnov S.V., Pestov A.V., Konovalov D.A. Adhesive Characteristics of Epoxy Glue in Relation to the Microgeometry of the Substrate Surface. In: AIP Conference Proceedings, 2018, 2053, 030066. Available at: https://doi.org/10.1063/1.5084427.

6. Oliver W.C., Pharr G.M. An improved technique for determining hardness and elastic modulus using load-displacement sensing indentation experiments. Journal of Materials Research, 1992, vol. 7, iss. 6, pp. 1564-1583. DOI: 10.1557/JMR.1992.1564.

7. Conradi M., Zorko M., Kocijan A., Verpoest I. Mechanical properties of epoxy composites reinforced with a low volume fraction of nanosilica fillers. Materials Chemistry and Physics, 2013, vol. 137, iss. 3, pp. 910-915. DOI: 10.1016/j.matchemphys.2012.11.00.

8. Morozov E.M., Zernin M.V. Kontaktnye zadachi mekhaniki razrusheniya [Contact Problems of Fracture Mechanics]. Moscow, Mashinostroenie Publ., 1999, 544 p. (In Russian).

9. Smirnov S.V., Veretennikova I.A., Vichuzhanin D.I. Modeling of delamination in multilayer metals produced by explosive welding under plastic deformation. Computational Continuum Mechanics, 2014, vol. 7, no. 4, pp. 398-411. DOI: 10.7242/1999-6691/2014.7.4.38. (In Russian).

10. Smirnov S.V., Domilovskaya T.V. Damage accumulation upon plastic deformation under monotonic loading. Russian Metallurgy (Metally), 2002, no. 5, pp. 470-476.

11. Smirnov S.V., Domilovskaya T.V. Definition of the kinetic equation form for damage under the plastic deformation. Fatigue and Fracture of Engineering Materials and Structures, 2003, vol. 26, iss. 4, pp. 373-379. DOI: 10.1046/j.1460-2695.2003.00624.x.

12. Kolmogorov V.L. Napryazheniya, deformatsii, razrushenie [Stresses, Strains, Fracture]. Moscow, Metallurgiya Publ., 1970, 232 p. (In Russian).

13. Hooke R. and Jeeves T.A. Direct Search Solution of Numerical and Statistical Problems. Journal of the ACM, vol. 8, no. 2, 1961, pp. 212-229. DOI: 10.1145/321062.321069. 
Подана в журнал: 23.10.2019

УДК 620.17:547-311

DOI: $10.17804 / 2410-9908.2019 .6 .026-036$

\title{
ОПРЕДЕЛЕНИЕ АДГЕЗИОННОЙ ПРОЧНОСТИ КЛЕЕВОГО СЛОИСТОГО МАТЕРИАЛА ПРИ ТРЕХТОЧЕЧНОМ ИЗГИБЕ
}

\author{
С. В. Смирнов ${ }^{1, \text { a) }}$, И. А. Веретенникова ${ }^{1, \text { б) }}$, Д. И. Вичужанин ${ }^{1, \text { в) }}$, А. В. Пестов ${ }^{2, \text { г) }}$ \\ ${ }^{1}$ Институт машиноведения Уральского отделения Российской академии наук, \\ ул. Комсомольская, 34, Екатеринбург, Российская Федерация \\ ${ }^{2}$ Институт органического синтеза им. И.Я. Постовского Уральского отделения Российской академии наук, \\ ул. Софьи Ковалевской, 22 , Екатеринбург, Российская Федерация \\ a) (D) https://orcid.org/0000-0002-2083-5377 @ svs@imach.uran.ru; \\ b) (D) https://orcid.org/0000-0002-8371-7546 $\otimes$ irincha@imach.uran.ru; \\ c) (iD https://orcid.org/0000-0002-6508-6859 mmm@imach.uran.ru; \\ d) (D) https://orcid.org/0000-0002-4270-3041 @ pestov@ios.uran.ru \\ "Ответственный автор. Электронная почта: svs@imach.uran.ru \\ Адрес для переписки: ул. Комсомольская, 34, г. Екатеринбург, 620049, Российская Федерация \\ Тел.: +7 (343) 374-40-76; факс: +7 (343) 374-53-30
}

В статье описаны методика и результаты исследования влияния напряженного состояния на адгезионную прочность в условиях сдвига для клеевого соединения. В качестве испытания выбран трехточечный изгиб многослойных образцов из алюминий-магниевого сплава АМг6-НВП и эпоксидного двухкомпонентного клея холодного отверждения. На основании анализа видеосъемки и конечно-элементного моделирования испытаний, была получена зависимость удельной работы адгезионного разрушения по механизму сдвига от показателя напряженного состояния. Показано, что при наличии растягивающих напряжений, нормальных к плоскости соединения, наблюдается низкая адгезионная прочность. В условиях действия сжимающих нормальных напряжений величина работы, затрачиваемой на адгезионное разрушение сдвигом, интенсивно возрастает. Полученные результаты могут быть использованы при оценке конструктивной прочности клеевых соединений в условиях сложного напряженного состояния.

Ключевые слова: адгезионное разрушение, клеевое соединение, эпоксидный клей, трехточечный изгиб.

\section{1. Введение}

Обеспечение требуемой адгезионной прочности соединения отдельных слоев является одной из важнейших задач при проектировании и эксплуатации изделий из слоистых материалов. Для ее оценки разработаны разнообразные методы испытаний, использование которых регламентировано национальными и отраслевыми стандартами, а для конкретных изделий - и техническими условиями. Наиболее распространенными методами испытаний для определения адгезионной прочности клеевых слоистых материалов являются испытания на сдвиг растяжением внахлест (ГОСТ 14759-69, ISO 4587-79, ASTM Standard D1002 - 10), на сдвиг в условиях сжатия [1], на растяжение компактных образцов для определения $\mathrm{K}_{1 \mathrm{c}}$ (ASTM Standard E399-90), на 4-точечный изгиб образца с надрезанным наружным слоем [2], изгиб со сдвигом (ГОСТ Р 57732-2017), на 3-точечный изгиб образцов (ГОСТ 25.604-82, ГОСТ P 56810-2015, ASTM Standard D790 - e2) и 3-точечный изгиб образца с межслойным продольным надрезом (ASTM Standard D7905/D7905M - 14). 
При эксплуатации конструкций, в состав которых входят элементы из клеевых слоистых материалов, может иметь место напряженно-деформированное состояние, характеризующееся наличием сдвигов на поверхностях клеевых соединений в условиях внешних сжимающих напряжений. Перечисленные выше методы испытаний, кроме трехточечного изгиба, не позволяют реализовать такую схему испытаний. Следует отметить, что ряд авторов не рекомендуют использовать результаты испытания на изгиб для оценки прочностных армированных пластиков из-за ограниченных возможностей корректной обработки результатов испытаний вследствие неоднородного напряженного состояния при изгибе [3-4]. По нашему мнению, влияние отмеченных недостатков можно нивелировать, используя современные методы мониторинга и анализа экспериментальных данных.

Цель статьи - продемонстрировать возможность использования испытаний на трехточечный изгиб для определения зависимости адгезионной прочности клеевых слоистых материалов от параметров напряженного состояния сдвига и действия сжимающих напряжений в направлении нормали к поверхности клеевого соединения. Разработанная методика позволяет расширить возможности прогноза адгезионной прочности в реальных условиях эксплуатации элементов конструкций из клеевых слоистых материалов в составе новых изделий или при продлении сроков эксплуатации технических объектов сверх гарантийных.

\section{2. Материалы и методики}

Испытывали слоистые образцы, состоящие из четырех пластин алюминиевого сплава АМг6-НВП, соединенных прослойками адгезива (рис. 1). Пластины размерами $80 \times 10 \times 2$ мм (длина $\times$ ширина $\times$ толщина) были изготовлены из элементов оболочечной конструкции летательного аппарата. Сплав АМг6-НВП представляет собой сплав АМг6 с химическим составом по ГОСТ 4784-97, но для увеличения прочности подвергнутый холодной пластической деформации прокаткой. Шероховатость Ra 0,8 мкм на контактных поверхностях пластин после механической и абразивной обработки была определена на бесконтактном профилометре-профилографе NT1100 и соответствует оптимальной для этого адгезива [5].

В качестве адгезива был использован эпоксидный двухкомпонентный клей холодного отверждения на основе коммерческой эпоксидной смолы ЭД-20 с эпоксидным числом $21,3 \%$, а в качестве отвердителя - коммерческий полиэтиленполиамин (ПЭПА). Массовое соотношение смола/отвердитель составляло 10:1. Отверждение смолы осуществляли при $25^{\circ} \mathrm{C}$ в течение 24 ч за счет реакции эпоксидных групп смолы с аминогруппами отвердителя. При отверждении смолы формируется сшитая структура, при этом степень конверсии эпоксидных групп составляет не более 20 \%, поскольку содержание гель-фракции составило 19 \%. Толщина слоев адгезива несколько отличалась, но в среднем составляла 1 мм.

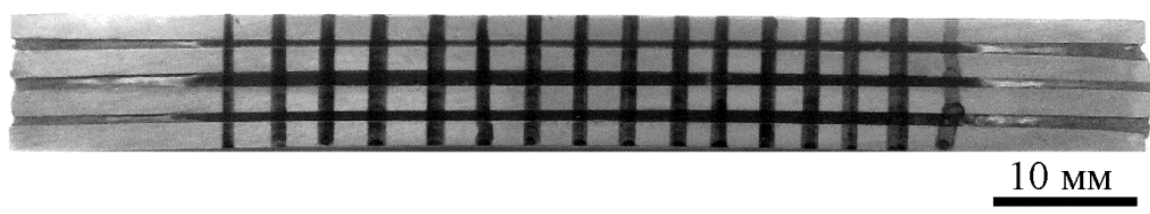

Рис. 1. Общий вид образца

Значения твердости $H=0,42$ ГПа и контактного модуля упругости $E^{*}=7,89$ ГПа адгезива определили методом инструментального индентирования с использованием системы для проведения микромеханических испытаний Fisherscope 2000xуm по методике ОливераФарра [6]. Для расчета значения нормального модуля упругости $E$ использовали формулу Герца [6]: 


$$
\frac{1}{E^{*}}=\frac{1-v^{2}}{E}+\frac{1-v_{\text {indenter }}^{2}}{E_{\text {indenter }}}
$$

где модуль Юнга $E_{\text {indenter }}=1140$ ГПа и коэффициент Пуассона $v_{\text {indenter }}=0,07$ для стандартного алмазного индентора, коэффициент Пуассона для эпоксидной смолы принимали равным $v=0,35[7]$.

По величине твердости, основываясь на рекомендациях [8], рассчитали значение напряжения $\sigma_{\mathrm{T}}=126$ МПа для адгезива на пределе текучести по соотношению $\sigma_{\mathrm{T}} \approx 0,3 \mathrm{H}$. Для осуществления возможности моделирования испытаний связь между степенью деформации $\varepsilon$ и интенсивность напряжений $\sigma$ на стадии неупругой деформации описывали билинейной функцией с коэффициентом упрочнения, имеющим малое значение, что практически соответствует не упрочняемому материалу:

$$
\sigma=126+0,1 \varepsilon
$$

Связь между накопленной деформацией и интенсивностью напряжений для сплава АМг6-НВП на стадии пластической деформации определяли по результатам растяжения стан-

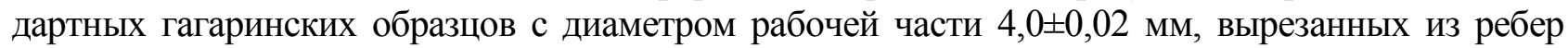
жесткости оболочки корпуса летательного аппарата. Испытания проводили на универсальной испытательной машине INSTRON 8801 со скоростью растяжения 1 мм/мин и для удобства моделирования в программном комплексе ANSYS аппроксимировали полилинейной функцией, которая представлена в табл. 1. Нормальный модуль упругости $E=7,1$ ГПа для материала пластин был рассчитан по результатам инструментального индентирования по формуле (1) при значении коэффициента Пуассона $v=0,32$, как это было описано выше для адгезива.

Таблица 1 - Полилинейная аппроксимация диаграммы деформационного упрочнения сплава АМг6-НВП

\begin{tabular}{|c|c|c|c|c|c|c|c|c|c|}
\hline$\varepsilon$ & 0,0044 & 0,083 & 0,0165 & 0,033 & 0,049 & 0,065 & 0,080 & 0,095 & 0,140 \\
\hline$\sigma$, МПа & 319 & 403 & 451 & 494 & 525 & 545 & 554 & 562 & 587 \\
\hline
\end{tabular}

Испытания на трехточечный изгиб проводили на универсальной серво-гидравлической испытательной машине Instron-8801 со скоростью перемещения плунжера 1 мм/мин. Фотографии образцов, размещенных на испытательной оснастке в рабочем пространстве машины до и после испытаний приведены на рис. 2. Расстояние между осями боковых опор составляло 80 мм. Диаметр средней цилиндрической и боковых опор составлял 10 мм. В процессе испытаний осуществлялось перемещение нижней траверсы машины с расположенным на ней боковыми опорами, верхний захват с центральной опорой оставался неподвижным. Прогиб образца в процессе испытаний измерялся с помощью датчика, входящего в состав измерительной системы машины и расположенного под средней частью образца (рис. 2). Величина максимального прогиба образца в процессе испытаний составляла 15,7 мм. Для изучения сдвига слоев адгезива относительно металлических пластин вследствие адгезионного разрушения на боковую поверхность образцов наносились фломастером реперные линии и в процессе испытаний проводилась видеосъемка с помощью видеокамеры бесконтактного оптического комплекса StrainMaster. Фиксация моментов адгезионного разрушения осуществлялась как начало визуально различимого относительного сдвига реперных линий на границе соединения адгезивных прослоек и пластин по результатам анализа последовательности кадров видеосьемки после проведения испытаний. На рис. 3 в качестве примера представлен 
один из кадров видеосъемки, на котором видны сдвиги реперных линий на границе между 2-м слоем адгезива и пластиной, считая от нижней поверхности образца. В табл. 2 представлены данные о четырех начальных сдвигах, установленных по результатам визуального анализа кадров видеосъемки в процессе испытаний.

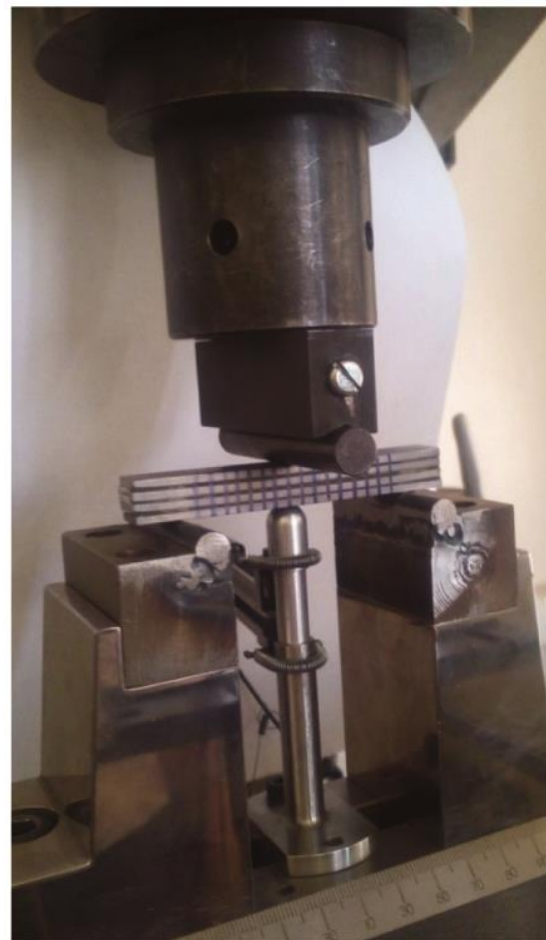

$a$

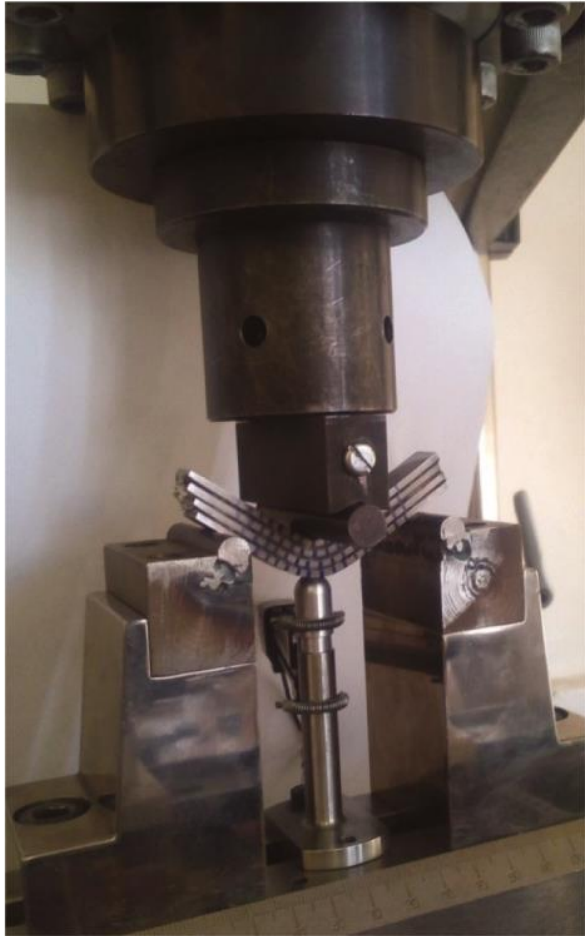

б

Рис. 2. Общий вид образца перед испытанием $(a)$ и под нагрузкой в конце испытания (б), под образцом установлен датчик для измерения прогиба

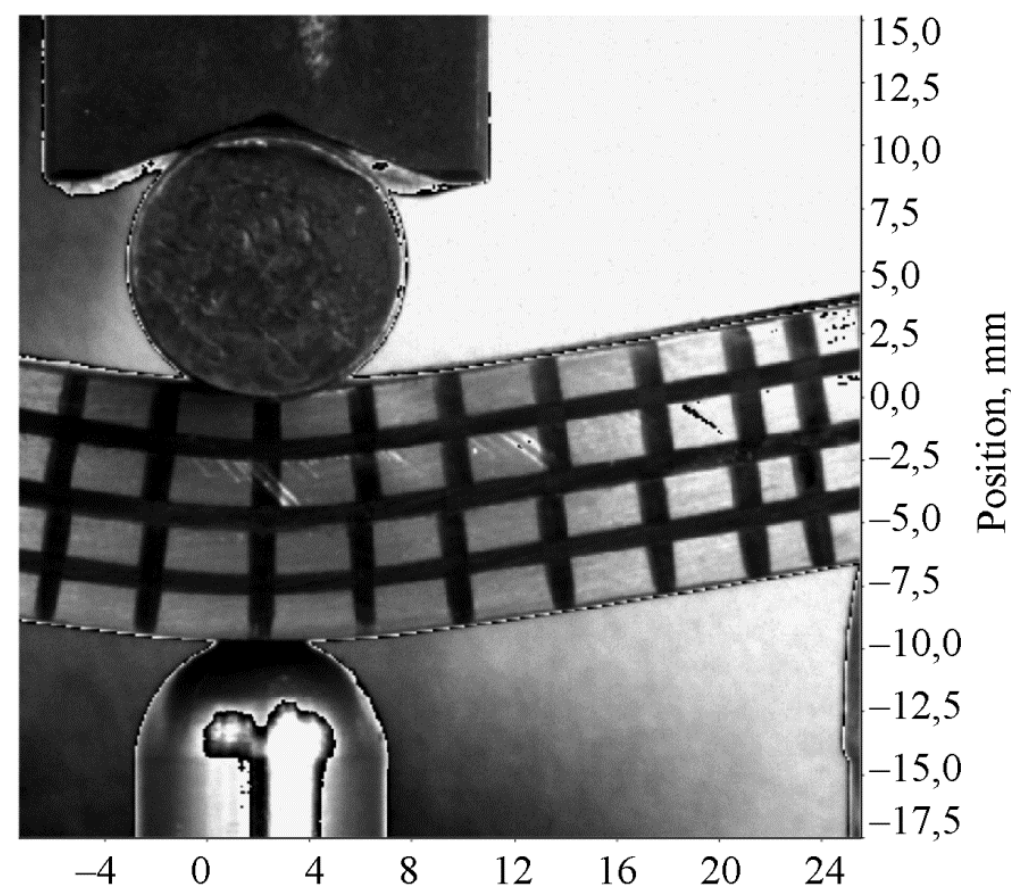

Position, mm

Рис. 3. Сдвиг реперных линий между 2-м слоем адгезива и 2-й алюминиевой пластиной

Determination of the adhesive strength of a laminated glue material under three-point bending / S. V. Smirnov, I. A. Veretennikova, D. I. Vichuzhanin, and A. V. Pestov // Diagnostics, Resource and Mechanics of materials and structures. 2019. - Iss. 6. - P. 26-36. - DOI: 10.17804/2410-9908.2019.6.026-036. 
Таблица 2 - Сведения о начальных сдвигах, зафиксированных при испытаниях

\begin{tabular}{|c|c|l|c|}
\hline $\begin{array}{c}\text { Номер } \\
\text { кадра }\end{array}$ & $\begin{array}{c}\text { Величина } \\
\text { прогиба, } \\
\text { мм }\end{array}$ & \multicolumn{1}{|c|}{ Местоположение начального сдвига } & $\begin{array}{c}\text { Номер шага } \\
\text { расчета при } \\
\text { моделировании }\end{array}$ \\
\hline 19 & 0,31 & $\begin{array}{l}\text { Нижняя граница 2-го снизу слоя адгезива } \\
\text { на 6-й реперной линии слева от центра }\end{array}$ & 19 \\
\hline 19 & 0,31 & $\begin{array}{l}\text { Нижняя граница 3-го снизу слоя адгезива } \\
\text { на 6-й реперной линии справа от центра }\end{array}$ & 23 \\
\hline 56 & 0,91 & $\begin{array}{l}\text { Нижняя граница 2-го снизу слоя адгезива } \\
\text { на 2-й реперной линии слева от центра }\end{array}$ & 197 \\
\hline 137 & 2,11 & $\begin{array}{l}\text { Нижняя граница 3-го снизу слоя адгезива } \\
\text { на 2-й реперной линии справа от центра }\end{array}$ & 237 \\
\hline
\end{tabular}

\section{3. Моделирование напряженно-деформированного состояния}

Для определения параметров напряженно-деформированного состояния, в местах наблюдаемого адгезионного разрушения и их изменения в процессе 3-хточечного изгиба было осуществлено моделирование испытаний с использованием программного комплекса ANSYS в ЦКП Института математики и механики УрО РАН. На рис. 4 приведена конечноэлементная сетка, использованная при моделировании испытаний.

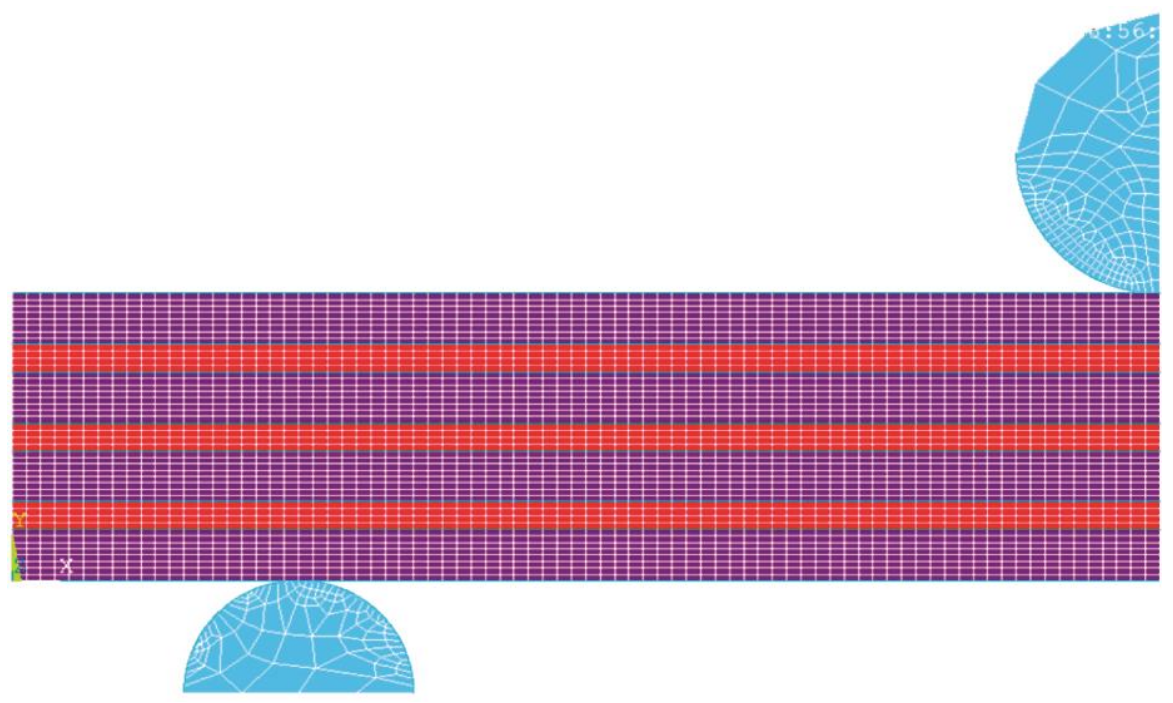

Рис. 4. Конечно-элементная сетка в исходном состоянии

В силу симметрии моделировали $\mathrm{S}$ схемы испытания. Граничные условия задавали в перемещениях. При постановке задачи использовали допущение о реализации плоскодеформированного состояния. Использовали 4-хузловые конечные элементы PLANE 2D. По толщине слоя адгезива было расположено 3, а по толщине металлической полосы 8 конечных элементов. Материал металлических пластин и адгезива представляли как упруго-пластическую изотропную, изотропно-упрочняющуюся среду с деформационным упрочнением и задавали в виде моделей полилинейно упрочняемого изотропного материала MISO (для АМг6-НВП) и билинейной (для адгезива) из перечня моделей ANSYS. Аппроксимации этих моделей, также как и упругие свойства, приведены в предыдущем разделе статьи. Материал стальных опор представляли как упругий с нормальным модулем упругости $E=200$ ГПа и коэффициентом Пуассона $v=0,3$. В первом приближении считали, что воз- 
никновение сдвига по механизму адгезионного разрушения в каждом слое образца происходит независимого от других слоев, что позволило считать их склеенными без возможности относительного смещения на границе соединения. На границах опор и образца задавали контактные конечные элементы с опцией surface-to-surface и коэффициентом трения по Амонтону-Кулону $\mu=0,25$ (сухое трение).

При вычислительной постановке использовали опцию large displacement и выбор peшателя program chosen. Начальный шаг по перемещению составлял $0,1 \%$ от конечного, при решении осуществлялся контроль по перемещениям и усилию, при отсутствии сходимости после 15 итераций автоматически в два раза уменьшался шаг по перемещению. При осуществленной постановке решение задачи было выполнено за 1250 шагов.

\section{4. Результаты и обсуждение}

Сопоставление результатов видеосъемки и моделирования позволило установить группу узлов конечно-элементной сетки, координаты которых в актуальной конфигурации соответствуют местоположению начальных относительных сдвигов реперных полос, а также номера шага расчета ближайших к моменту видиофиксации сдвигов (табл 2). Так как в соответствии с вычислительной постановкой задачи узлы конечно-элементной сетки на границе слоев образца являются общими для адгезива и пластин, то для большей определенности при расчетах удельной работы рассматривалось напряженно-деформированное состояние в узлах сетки, ближайших от границы соединения, по три со стороны адгезива и пластины. Расчет необходимых для обработки результатов экспериментов характеристик НДС осуществлялся, используя результаты моделирования. Для этого с помощью соответствующей опции программы ANSYS для выделенных узлов были пошагово выгружены значения компонент тензоров напряжений и деформаций, что позволило восстановить историю изменения напряженно-деформированного состояния от начала испытания до моментов наблюдения начальных относительных сдвигов реперных линий, классифицированных как локальное адгезионное разрушение по механизму сдвига.

Локальную удельную работу адгезионного разрушения по механизму сдвига $W_{t}^{*}$ рассчитывали как сумму полных удельных (упругая $W_{e}+$ пластическая $W_{p}$ ) работ, рассчитанных для пар ближайших узлов конечно-элементной сетки, расположенных симметрично относительно границы соединения со стороны адгезива и металлической пластины:

$$
W_{t}^{*}=\sum_{i=1}^{N} \sigma_{i} \Delta \varepsilon_{t i} h_{i}
$$

где $\sigma_{i}$ и $\Delta \varepsilon_{t i}-$ интенсивность напряжений и приращение тангенциальной деформации на $i$-шаге расчета, которое определяется как сумма приращений упругой и пластической деформации; $h_{i}$ - толщина слоя конечных элементов, равная размеру конечного элемента в направлении нормали к границе соединения на $i$-шаге расчета со стороны адгезива и пластин соответственно. В нашем случае в исходном состоянии значения $h=0,33$ мм и для адгезива, и для пластин. Изменение $h_{i}$ в процессе деформирования рассчитывали по изменению координат узлов конечно-элементной сетки.

В соответствии с результатами ранее проведенных исследований [9] будем считать, что сопротивление адгезионному разрушению зависит от показателя напряженного состояния

$$
k=\frac{\sigma_{n}}{\sigma_{T}},
$$

где $\sigma_{n}$ - нормальное напряжение к площадке на границе соединения адгезива. 
Угол $\alpha_{y}$ наклона вектора нормали площадки и направляющие косинусы $n_{x}, n_{y}$ рассчитывали по формуле:

$$
\alpha_{y}=\operatorname{arctg}\left(\frac{u_{y 2}-u_{y 1}}{\left(x_{2}^{0}-x_{1}^{0}\right)+\left(u_{x 2}-u_{x 1}\right)}\right) ; n_{y}=\cos \alpha_{y} ; n_{x}=\sin \alpha_{y},
$$

где $x_{1}^{0}, x_{2}^{0}$ - координаты соседних выделенных узлов сетки в исходном до деформации состоянии; $u_{x 1}, u_{x 2}, u_{y 1}, u_{y 2}$ - перемещения этих узлов при деформировании. Зависимость $W_{t}^{*}(k)$ по аналогии с зависимостью предельной деформации адгезионного разрушения $\varepsilon_{t}^{*}(k)$, использованной в работе [9], искали в виде экспоненты:

$$
W_{t}^{*}(k)=a_{0} \exp \left(-a_{1} k\right),
$$

где $a_{0}$ и $a_{1}-$ эмпирические коэффициенты.

В соответствии концепциями, заложенными в модели механики поврежденности, разрушение является не одноактным катастрофическим явлением, а представляет собой процесс накопления микроскопических дефектов, которые возникают и развиваются в соответствии с некоторыми закономерностями, а после достижения их плотности предельного значения происходит возникновение разрушающей трещины. Существует достаточно большое количество моделей разрушения, отличающихся тем, какой параметр берется в качестве определяющего приращения поврежденности (плотность, деформация, энергия и др.) и какой закон накопления поврежденностей (линейный, степенной, экспоненциальный и др.). Ранее было показано $[10,11]$, что при простом и близком к нему монотонном нагружении металлических материалов без нагрева и в условиях умеренных скоростей адекватные результаты достигаются при использовании линейной деформационной модели поврежденности В.Л. Колмогорова [12]. Для адгезивных материалов подобные исследования не известны, поэтому будем также использовать линейную модель накопления поврежденности $\omega$, которая для использования при численных расчетах имеет вид

$$
\omega=\sum_{i=1}^{N} \frac{\Delta W_{t i}}{W_{t}^{*}\left(k_{i}\right)}
$$

До начала нагружения поврежденность $\omega=0$. В момент возникновения трещины $\omega=1$. Неизвестные коэффициенты функции (6) определяли, используя процедуру идентификации по критерию наилучшего выполнения условия адгезионного разрушения при расчетах по формуле (7).

Рассчитав величину поврежденности для каждого указанного в табл. 2 случая адгезионного разрушения, по формуле (3) осуществляли минимизацию функционала квадратичных отклонений рассчитанной поврежденности от 1:

$$
\sum_{j=1}^{4}\left(1-\omega_{j}\right)^{2} \rightarrow \min
$$

где $j$ - номер испытания. Минимизацию функционала (8) выполнили путем варьирования неизвестных коэффициентов, входящих в функцию $W_{t}^{*}(k)$ и соответственно в формулу (6), методом Хука-Дживса [13]. Для того чтобы не попасть в локальный минимум функционала (6), процедура Хука-Дживса была выполнена не для одной начальной точки поиска, а для неко- 
торого их множества. В результате были определены значения эмпирических коэффициентов в экспоненциальной зависимости (6): $a_{1}=0,575 ; a_{2}=80,27$.

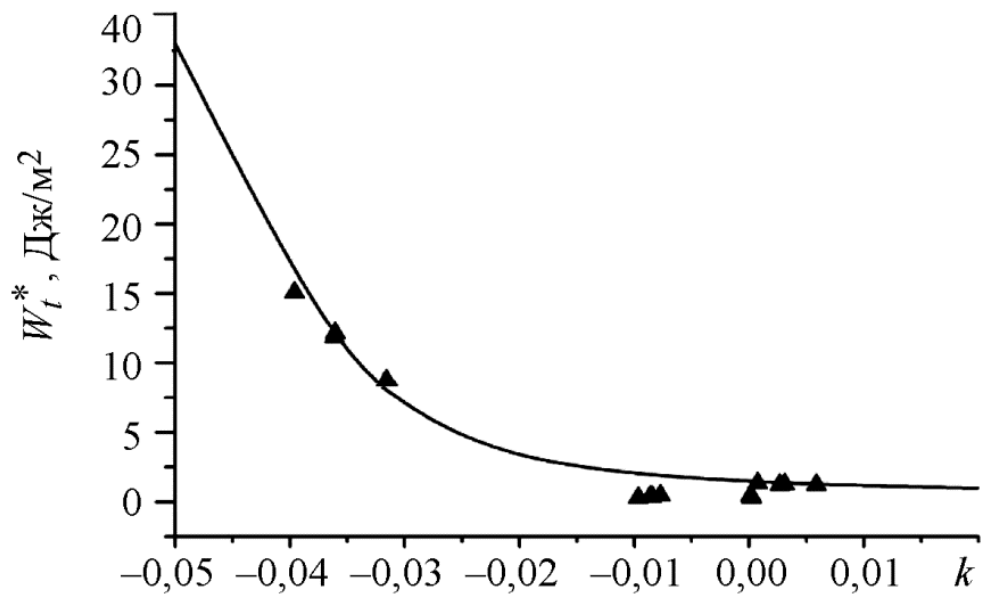

Рис. 5. Диаграмма предельной удельной работы адгезионного разрушения сдвигом

На рис. 5 приведена диаграмма предельной удельной работы адгезионного разрушения по механизму сдвига для исследованного адгезива. Видно, что при наличии растягивающих напряжений нормальных к плоскости клеевого соединения $(k>0)$ величина $W_{t}^{*}(k)$ мала, что свидетельствует о низкой адгезионной прочности сдвигу. В то же время в условиях действия сжимающих нормальных напряжений $(k<0)$ величина работы, затрачиваемой на адгезионное разрушение сдвигом, интенсивно возрастает.

\section{5. Заключение}

Выполнено исследование адгезионного разрушения при испытаниях на 3-хточечный изгиб клеевого соединения пластин из алюминий-магниевого сплава АМг6-НВП с помощью эпоксидного двухкомпонентного клея холодного отверждения на основе коммерческой эпоксидной смолы ЭД-20, отвержденной полиэтиленполиамином. Используя результаты видеосъемки деформирования образцов и результаты конечно-элементного моделирования испытаний, была получена функциональная зависимость удельной работы $W_{t}^{*}(k)$ адгезионного разрушения по механизму сдвига от показателя напряженного состояния $k$, которая описывается экспоненциальным законом. Установлено, что при наличии растягивающих напряжений, нормальных к плоскости клеевого соединения $(k>0)$, величина $W_{t}^{*}(k)$ мала, что свидетельствует о низкой адгезионной прочности сдвигу. В то же время в условиях действия сжимающих нормальных напряжений $(k<0)$ величина работы, затрачиваемой на адгезионное разрушение сдвигом, интенсивно возрастает. Полученные результаты могут быть использованы при оценке конструктивной прочности клеевых соединений в условиях сложного напряженного состояния.

\section{Благодарность}

Разработка экспериментальных методик исследования полимерных покрытий выполнена в соответствии с планом работ ИМАШ УрО РАН по теме АААА-А18-118020790145-0. Уральским отделением РАН была оказана финансовая поддержка в рамках проекта 18-11-1-11 в приобретении материалов для испьтаний и в исследовании методом трехточечного изгиба влияния напряюенного состояния на удельную работу адгезионного разрушения по механизму 
сдвига для клеевого соединения. При выполнении экспериментальных исследований было использовано оборудование ЦКП «Пластометрия» ИМАШ УрО РАН.

\section{Литература}

1. Hutchinson J. W., Suo Z. Mixed Mode Cracking in Layered Materials // Advanced in Applied Mechanics. - 1992. - Vol. 29. - P. 63-191. - DOI: 10.1016/S0065-2156(08)70164-9.

2. Volinsky A. A., Moody N. R., Gerberich W. W. Interfacial toughness measurements for thin films on substrates // Acta Materialia. - 2002. - Vol. 50. - P. 441-466. DOI: 10.1016/S1359-6454(01)00354-8.

3. Тамуж В. П., Протасов В. Д. Разрушение конструкций из композитных материалов. Рига : Зинатне, 1986. - 264 с.

4. Тарнопольский Ю. М., Кинцис Т. Я. Методы статических испытаний армированных пластиков. - М. : Химия, 1981. - 272 с.

5. Adhesive Characteristics of Epoxy Glue in Relation to the Microgeometry of the Substrate Surface / E. O. Smirnova, I. A. Veretennikova, S. V. Smirnov, A. V. Pestov, D. A. Konovalov // AIP Conference Proceedings. - 2018. - Vol. 2053. - 030066. - DOI: 10.1063/1.5084427.

6. Oliver W. C., Pharr G. M. An improved technique for determining hardness and elastic modulus using load-displacement sensing indentation experiments // Journal of Materials Research. 1992. - Vol. 7, iss. 6. - P. 1564-1583. - DOI: 10.1557/JMR.1992.1564.

7. Mechanical properties of epoxy composites reinforced with a low volume fraction of nanosilica fillers / M. Conradi, M. Zorko, A. Kocijan, I. Verpoest // Materials Chemistry and Physics. - 2013. - Vol. 137, iss. 3. - P. 910-915. - DOI: 10.1016/j.matchemphys.2012.11.00.

8. Морозов Е. М., Зернин М. В. Контактные задачи механики разрушения. - М. : Машиностроение, 1999. - 544 с.

9. Смирнов С. В., Веретенникова И. А., Вичужанин Д. И. Моделирование расслоения при пластической деформации биметаллического материала, полученного сваркой взрывом // Вычислительная механика сплошных сред. - 2014. - Т. 7, № 4. - С. 398-411. DOI: $10.7242 / 1999-6691 / 2014.7 .4 .38$.

10. Смирнов С. В., Домиловская Т. В. Накопление поврежденности при пластической деформации в условиях монотонного нагружения // Металлы. - 2002. - № 5. - С. 68-76.

11. Smirnov S. V., Domilovskaya T. V. Definition of the kinetic equation form for damage under the plastic deformation // Fatigue and Fracture of Engineering Materials and Structures. 2003. - Vol. 26, iss. 4. - P. 373-379. - DOI: 10.1046/j.1460-2695.2003.00624.x.

12. Колмогоров В. Л. Напряжения, деформации, разрушение. - М. : Металлургия, 1970. $232 \mathrm{c}$.

13. Хук Р., Дживс Т. А. Прямой поиск решения для числовых и статических проблем. 1961. - C. 212-219. 